\title{
ON THE SINGULAR BOUNDARY VALUE PROBLEM FOR ELLIPTIC EQUATIONS
}

\author{
BY
}

\section{KAZUNARI HAYASHIDA}

ABSTRACT. The operator $\mathcal{Q}$ is elliptic and of second order in a domain $Q$ in $R^{N}$. We consider the following boundary value problem: $\mathcal{L} u=f$ in $Q$ and $\$ u=0$ on $\partial \Omega$, where $\$=a d / d n+\beta(d / d n$ is the conormal derivative on $\partial \Omega)$.

The coefficient $a$ is assumed to be nonnegative. However, $\alpha$ may vanish partly on $\partial \Omega$. Then the regularity of the weak solutions for the above problem is shown by the variational method.

1. We denote by $\Omega$ a domain in the $N$-dimensional Euclidean space $R^{N}$ with the boundary $\partial \Omega$ of class $C^{\infty}$ and the closure $\bar{\Omega}$. Let us assume that $\Omega$ is bounded.

Consider an elliptic operator $\mathcal{L}$ of second order in $\bar{\Omega}$ :

$$
\mathcal{L}=-\frac{\partial}{\partial x_{j}}\left(a_{i j} \frac{\partial}{\partial x_{i}}\right)+b_{i} \frac{\partial}{\partial x_{i}}+c,
$$

where all the coefficients are real and sufficiently smooth. We assume that $\left(a_{i j}\right)$ is uniformly positive definite in $\Omega$, and there is a sufficiently large number $c_{0}$ satisfying $c \geq c_{0}$ in $\Omega$. The boundary operator will be taken as

$$
\mathfrak{B}=\alpha \frac{d}{d n}+\beta,
$$

where

$$
\frac{d}{d n}=a_{i j} \cos \left(\nu, x_{j}\right) \frac{\partial}{\partial x_{i}}
$$

and $\alpha, \beta$ are real and sufficiently smooth on $\partial \Omega$.

In this paper we consider the following boundary value problem:

$$
\mathfrak{L}_{u=f} \text { in } \boldsymbol{\Omega} \text { and } \mathfrak{B}_{u}=0 \text { on } \partial \Omega \text {. }
$$

Received by the editors August 24, 1972 and, in revised form, February 3, 1973. AMS (MOS) subject classifications (1970). Primary 35J20, 35J 25.

Key words and phrases. Elliptic operator, boundary operator, regularity problem, elliptic regularization, Sobolev space, variational method.

(1) The letter $\nu$ is the outer normal on $\partial \Omega$. 
If $\alpha$ vanishes identically and $\beta$ does not vanish, the problem is nothing else but the Dirichlet problem which was solved by many authors (cf., e.g., [7]). When $a$ $\neq 0$ on $\partial \Omega$, the problem is reduced to the boundary value problem of the third kind which was also solved by many authors (cf., e.g., [4]). However, if a vanishes on some portion of $\partial \Omega$, the boundary value problem (1.3) seems to be not so easy. In particular, Itô [3] constructed the Green function and solved (1.3) for the case when $\alpha, \beta \geq 0$ and $\alpha+\beta \equiv 1$. His tool is the construction of the Green function for the nonstationary state by integral equations. Now the following question arises:

Can the problem (1.3) be solved by the tool of functional analysis (or by variational methods)?(2)

On the other hand, recently Maz' ${ }^{\prime} \mathrm{a}$ [6] has considered the following boundary value problem

$$
\mathfrak{L}_{u=0} \text { and }\left(\alpha_{i} \frac{\partial}{\partial x_{i}}+\beta\right) u=g \text { on } \partial \Omega,
$$

where the assumption on $\left\{\alpha_{i}\right\}$ permits the case of $\alpha_{i}=0$ on some portion of $\partial \Omega$. He has proved the existence and the uniqueness of weak solutions for $\left(1.3^{\prime}\right)$ by the variational method. However the regularity of the solutions has not been shown in [6]. Thus the regularity problem for $\left(1.3^{\prime}\right)$ arises. For (1.3) the regularity is known in the classical sense by the result of Ito [3]. But it seems to us that the regularity problem for (1.3) is still open in the sense of functional analysis (or of function spaces).

Thus it seems to be meaningful to show the existence and the uniqueness of sufficiently smooth solutions for (1.3) under some assumptions for $\alpha$ and $\beta$ (see (A) in $\$ 2$ ). The method of reducing the problem (1.3) to a degenerate variational elliptic problem is due to [6] (see the beginning of \$4). And our argument is based upon the elliptic regularization. Particularly we have referred to the work of Baouendi and Goulaouic [1].

The author expresses his hearty thanks to Professor A. Takeshita for his helpful advice and incessant encouragement. The author wishes to thank Professor Y. Kato for his useful discussions and kind advice.

2. Let the operator $\mathcal{K}$ be such that

$$
\mathcal{K}=a_{i j} \frac{\partial^{2}}{\partial x_{i} \partial x_{j}}+\left(\left(a_{i j}\right)_{x_{i}}+b_{j}\right) \frac{\partial}{\partial x_{j}},(3)
$$

and consider the function $\Phi$ satisfying

(2) The question was suggested by Professor S. Mizohata.

(3) We denote $\partial() / \partial x_{i}$ by ()$_{x_{i}}$ simply. 


$$
K \Phi=0 \text { in } \Omega, \quad \Phi=\alpha(x) \text { on } \partial \Omega \text {. }
$$

The existence of such a function $\Phi$ is well known, and $\Phi$ is sufficiently smooth in $\bar{\Omega}$. Secondly put

$$
\gamma=\frac{1}{2} \cos \left(\nu, x_{i}\right)\left(a_{i j} \Phi_{x_{j}}+b_{i} \Phi\right)+\beta
$$

Then we impose the following assumption on $\alpha$ and $\beta$ :

$$
\text { (i) } a \neq 0 \text { and } a \geq 0 \text { on } \partial \Omega \text {. }
$$

(ii) $\gamma>0$ on $\partial \Omega$.

From (i) of the assumption we see that $\Phi>0$ in $\Omega$ (see p. 326 in [2]).

Now we shall prepare some function spaces. First for a nonnegative integer $k$ we put

$$
(u, v)_{k}=\sum_{|\alpha| \leq k} \int_{\Omega} D^{\alpha} u \cdot D^{\alpha} v d x,\left({ }^{4}\right) \quad\|u\|_{k}^{2}=(u, u)_{k}
$$

and

$$
\dot{(} u, v)=(u, v)_{0}, \quad\|u\|=\|u\|_{0} .
$$

Let us denote by $H^{k}(\Omega)$ the Sobolev space with its norm \|\|$_{k}$. For nonnegative real $k$ the norm \|\|$_{k}$ and the space $H^{k}(\Omega)$ are defined in the usual way respectively.

Next we put by $V(\Omega)$ and $W(\Omega)$ the completion of $C^{\infty}(\bar{\Omega})$ with respect to the following norms, respectively:

$$
\|u\|_{V}=\sum_{i}\left\|\Phi^{1 / 2} u_{x_{i}}\right\|+\left\|\Phi^{1 / 2} u\right\|
$$

and

$$
\|u\|_{W}=\|u\|_{V}+\left(\int_{\partial \Omega} u^{2} d \sigma\right)^{1 / 2} \cdot\left({ }^{5}\right)
$$

Further we define the following norm for integers $k$ :

$$
\|u\|_{k}=\|u\|_{k-1}+\sum_{|a| \leq k}\left\|\Phi^{1 / 2} D^{a} u\right\| \quad(k \geq 1)
$$

and

$$
\|u\|_{0}=\left\|\Phi^{1 / 2} u\right\| \cdot
$$

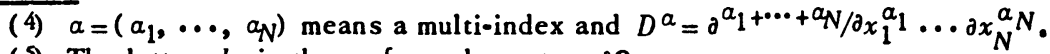

(5) The letter $d \sigma$ is the surface element on $\partial \Omega$. 
Let us denote by $V^{k}(\Omega)$ the completion of $C^{\infty}(\bar{\Omega})$ with respect to the norm \|\|$\|_{k}$. From Lemma 3.4 (in $\S 3$ ) it will be seen that $V^{1}(\Omega)=V(\Omega)$.

Remark 1. The above spaces are Hilbert spaces with weighted norms. Recently the weighted norm has been applied to the study of degenerate elliptic equations (cf., e.g., [1], [5], [8], [9]).

Our main theorem is as follows:

Theorem. For any given $f \in V^{k}(\Omega)(k \geq 0)$ there is a unique solution $u \epsilon$ $V^{k+2}(\Omega)$ satisfying (1.3).

Remark 2. From the theorem and Sobolev's lemma we have immed iately the following statement: Suppose that the coefficients in (1.1) and (1.2) are all in $C^{\infty}(\bar{\Omega})$. Then for any $f \in C^{\infty}(\bar{\Omega})$ there exists a unique solution $u \in C^{\infty}(\bar{\Omega})$ of the boundary value problem (1.3). This is nothing else but the result of Itô [3]. His proof is due to the construction of the Green function for the nonstationary state by integral equations.

3. In this section we shall prepare some properties for the function $\Phi$ defined in (2.1). Let $P_{0}$ be a fixed point on $\partial \Omega$ satisfying $\alpha\left(P_{0}\right)=0$ and $U\left(P_{0}\right)$ be a sufficiently small neighborhood of $P_{0}$. The sets $\bar{\Omega} \cap U\left(P_{0}\right), \partial \Omega \cap U\left(P_{0}\right)$ and the point $P_{0}$ can be mapped in a one-to-one $C^{\infty}$ way into $\left\{\left(x_{1}, \ldots, x_{N}\right) \mid x_{N} \geq 0\right\}$, $\left\{\left(x_{1}, \ldots, x_{N}\right) \mid x_{N}=0\right\}$ and the origin, respectively. From now on we put

$$
x=\left(x_{1}, \cdots, x_{N-1}\right), \quad y=x_{N} .
$$

Then we can write as $\left(x_{1}, \ldots, x_{N}\right)=(x, y)$ simply. Hereafter the transformed functions in the new coordinate will be denoted also by the original notation.

First we see

Lemma 3.1. There is a constant $C$ such that in a neigbborbood of the origin it bolds

$$
y \leq C \Phi(x, y) \quad(y \geq 0)
$$

Proof. The function $\Phi$ satisfies (3.1) in the original coordinate and $\Phi>0$ in $y>0$. Hence by the well-known maximum principle (see p. 328 in [2]) it follows that $\partial \Phi / \partial y>0$ at $\left(x^{0}, 0\right)$, if $\Phi\left(x^{0}, 0\right)=0$. Of course, this inequality holds also in the neighborhood of $\left(x^{0}, 0\right)$. On the other hand, we easily see $(3.1)$ if $\Phi\left(x^{0}, 0\right)$ $>0$. Thus we have obtained (3.1).

Observing $\Phi$ on $y=0$, we have the following

Lemma 3.2. In a ne igbborbood of the origin it bolds

$$
\left(D_{x} \alpha\right)^{2} \leq C a \quad(y=0)
$$


where $C$ is a constant and $D_{x}$ means some first derivatives $\partial / \partial x_{i}(i=1, \ldots$, $N-1)$.

Proof.(6) First we consider the case of $N=2$. And for a sufficiently small $a>0$, let us consider $a$ in the open interval $(-a, a)$. We denote by $a^{\prime}$ the first derivative of $a$. If $a^{\prime} \neq 0$ in $(-a, a)$, then $a$ is strictly monotone in $(-a, a)$. Hence (3.2) follows in $(-a / 2, a / 2)$. Thus we may assume $a^{\prime}$ vanishes somewhere. For any fixed $x$ let $x^{\prime}$ be such that $\alpha^{\prime}\left(x^{\prime}\right)=0$ and $\alpha^{\prime} \neq 0$ in $\left(x^{\prime}, x\right)$ (or $\left.\left(x, x^{\prime}\right)\right)$. Then by the well-known theorem we see

$$
\alpha^{\prime}(x)^{2} /\left(\alpha(x)-\alpha\left(x^{\prime}\right)\right)=2 \alpha^{\prime}(\xi) a^{\prime \prime}(\xi) / \alpha^{\prime}(\xi),
$$

where $\xi$ is some point in $\left(x^{\prime}, x\right)$. Therefore it follows that

$$
a^{\prime}(x)^{2} \leq 2\left|\alpha^{\prime \prime}(\xi)\right|\left|\alpha(x)-a\left(x^{\prime}\right)\right| \leq 2\left|\alpha^{\prime \prime}(\xi)\right| \alpha(x) .
$$

This implies (3.2) immediately. For a general $N$ we put

$$
C=2 \max _{1 \leq i \leq N}\left|\frac{\partial^{2} a}{\partial x_{i}^{2}}\right| .
$$

Then from the case of $N=2$ we easily see that the constant $C$ is required in (3.2).

Further for $\Phi$ we also have

Lemma 3.3. There is a constant $C$ satisfying

$$
\left(D_{x} \Phi\right)^{2} \leq C \Phi \quad(y \geq 0)
$$

in a ne igbborbood of the origin.

Proof. We may assume that $\Phi=0$ at the origin. By Taylor expansion,

$$
\Phi(x, y)=\alpha(x)+y\left(D_{y} \Phi\right)(x, \theta y) \quad(0<\theta<1) .
$$

Putting $\psi(x, y)=\left(D_{y} \Phi\right)(x, \theta y)$, we see

$$
D_{x} \Phi(x, y)=D_{x} a(x)+y D_{x} \psi(x, y) .
$$

From the proof of Lemma 3.1, $\psi(x, 0)>0$ holds.

Now it is easily seen that

(6) The idea of the proof is due to Professor K. Nanba. 


$$
\left(D_{x} \Phi\right)^{2} / \Phi \leq\left(\frac{D_{x} a}{a^{1 / 2}}+\frac{y}{a^{1 / 2}} D_{x} \psi\right)^{2} /\left(1+\frac{y}{a} \psi\right) .
$$

We shall show that the right-hand side of this inequality is bounded when $\left(x^{n}, y^{n}\right)$ $\rightarrow\left(x^{0}, 0\right)$. By Lemma 3.2 it is obvious that $D_{x} a / a^{1 / 2}$ is bounded. When $\left(x^{n}, y^{n}\right)$ $\rightarrow\left(x^{0}, 0\right)$, the behavior of $y / a$ is classified into two cases:

(i) $y / a \rightarrow+\infty$ or

(ii) $y / \alpha$ is bounded.

In the case (i) the right-hand side of (3.4) tends to 0 obviously. For the case (ii) the right-hand side of (3.4) is bounded, since $y / a^{1 / 2}$ is bounded. Hence (3.3) has been shown.

The following lemma is analogous to the interpolation inequality obtained by Baouendi and Goulaouic [1].

Lemma 3.4. For any $\delta>0$ and any $u \in C^{\infty}\left(\bar{R}_{+}^{N}\right)(7)$ with compact support,

$$
\|u\|^{2} \leq C\left(\delta\left\|\Phi^{1 / 2} u_{y}\right\|^{2}+\delta^{-1}\left\|\Phi^{1 / 2} u\right\|^{2}\right)
$$

where $C$ is a constant independent of $\delta$ and $u$.

Proof. We proceed in parallel with [1]. Obviously,

$$
u(x, y)^{2}=-2 \int_{y}^{\infty} u u_{y} d y \quad(y \geq 0)
$$

Denoting the right-hand side by $F(y)$, we see

$$
\int_{0}^{\infty} u(x, y)^{2} d y=\int_{0}^{\infty} F(y) d y=[y F(y)]_{0}^{\infty}-\int_{0}^{\infty} y F^{\prime}(y) d y=-2 \int_{0}^{\infty} y u u_{y} d y .
$$

Hence Cauchy's inequality yields

$$
\int_{0}^{\infty} u^{2} d y \leq \delta \int_{0}^{\infty} y u_{y}^{2} d y+\delta^{-1} \int_{0}^{\infty} y u^{2} d y
$$

Integrating by $x$, we obtain

$$
\|u\|^{2} \leq \delta\left\|y^{1 / 2} u_{y}\right\|^{2}+\delta^{-1}\left\|y^{1 / 2} u\right\|^{2} .
$$

Applying Lemma 3.1 to the right-hand side, we have completed the proof.

4. Again we shall consider the original coordinate. For $u, v \in C^{1}(\bar{\Omega})$ let us set

$$
B[u, v]=\left(a_{i j} \Phi u_{x_{i}}, v_{x_{j}}\right)+\left(\left(a_{i j} \Phi_{x_{j}}+b_{i} \Phi\right)_{x_{x_{i}}}, v\right)+(\Phi c u, v)+\int_{\partial \Omega} \beta u v d \sigma
$$

where $d \sigma$ is a surface element on $\partial \Omega$. Such a definition of $B[u, v]$ is based on the idea of $\mathrm{Maz}^{\prime} \mathrm{ja}[6]$. If for any $v \in C^{1}(\bar{\Omega}), B[u, v]=(\Phi /, v)$, then we have (1.3)

( 7 We denote by $C^{\infty}\left(\bar{R}_{+}^{N}\right)$ the set of functions which belongs to $C^{\infty}$ class in $y \geq 0$. 
(in the weak sense) by integration by parts. That is, our problem is reduced to the degenerate elliptic equations.

Now by integration by parts and $(2.1)(K \Phi=0)$, we see, for $u \in C^{1}(\bar{\Omega})$,

$$
B[u, u]=\left(a_{i j} \Phi u_{x_{i}}, u_{x_{j}}\right)+\left(\left(c-1 / 2\left(b_{i}\right)_{x_{i}}\right) \Phi u, u\right)+\int_{\partial \mathbf{\Omega}} \gamma u^{2} d \sigma,
$$

where $\gamma$ is the same constant as in (2.2). From our assumption (A) in $\$ 2, \gamma$ is positive. Hence we have

$$
\|u\|_{W}^{2} \leq C B[u, u] \quad\left(u \in C^{1}(\bar{\Omega})\right) .
$$

For any $\epsilon>0$ let us define the bilinear form $B[u, v]$ on $H^{1}(\Omega)$ in such a way that $B[u, v]=\epsilon(u, v)_{1}+B[u, v]$. Obviously

$$
\left|B_{\epsilon}[u, v]\right| \leq C\|u\|_{1}\|v\|_{1} .
$$

From (4.1) we see

$$
\epsilon\|u\|_{1}^{2}+\|u\|_{W}^{2} \leq C B_{\epsilon}[u, u]
$$

where $C$ is independent of $\epsilon$.

Let us take a positive sequence $\left\{\epsilon_{\nu}\right\}$ of positive numbers such as $\epsilon_{\nu} \rightarrow 0$. For simplicity we put $B_{\nu}[u, v]=B_{\epsilon}[u, v]$. For a given $f \in V^{0}(\Omega)$ let us take a sequence $\left\{f_{\nu}\right\}$ in $C^{\infty}(\bar{\Omega})$ satisfying $f_{\nu} \rightarrow f(\nu \rightarrow \infty)$ in the topology of $V^{0}(\Omega)$. By (4.2) and (4.3) the theorem of Lax-Milgram can be applied to $B_{\nu}[u, v]$. Hence there is a unique $u_{\nu} \in H^{1}(\Omega)$ satisfying

$$
B_{\nu}\left[u_{\nu}, v\right]=\left(\Phi f_{\nu}, v\right) \text { for any } v \in H^{1}(\Omega) \text {. }
$$

By the well-known fact, $u_{\nu}$ is sufficiently smooth in $\bar{\Omega}$. In particular, substituting $v=u_{\nu}$ into (4.4), we have by (4.3) and Schwarz' inequality

$$
\epsilon_{\nu}\left\|u_{\nu}\right\|_{1}^{2}+\left\|u_{\nu}\right\|_{W}^{2} \leq C\left\|f_{\nu}\right\|_{0}\left\|u_{\nu}\right\|_{W} .
$$

Therefore we have obtained the following

Proposition 4.1. For the solutions $u_{\nu}$ in (4.4) the functions $\epsilon_{\nu}^{1 / 2}\left\|u_{\nu}\right\|_{1}$ and $\left\|u_{\nu}\right\|_{W}$ are bounded with respect to $\nu$.

From this proposition we see that there is a subsequence of $\left\{u_{\nu}\right\}$ which converges to $u$ weakly in $W$. In the next section we shall show that $\left\|u_{\nu}\right\|_{1}$ is bounded. If this is established, then $u$ is in $H^{1}(\Omega)$ and by (4.4) we have $B[u, v]$ $=(\Phi f, v)$ for any $v \in H^{1}(\Omega)$. This equality means that $u$ is the required weak solution of (1.3). 
5. Our aim in this section is to prove the following

Proposition 5.1. For solutions $u_{\nu}$ of (4.4) the quantities $\left\|u_{\nu}\right\|_{2}$ are bounded with respect to $\nu$. That is, our main theorem in $\$ 2$ bolds for $k=0$.

Before proving the proposition we prepare some lemmas. Let $P_{0}$ be a fixed point on $\partial \Omega$ satisfying $\alpha\left(P_{0}\right)=0$ and $B_{r}\left(P_{0}\right)$ be a sphere with its center $P_{0}$ and with its radius $r$. Taking $r_{0}$ sufficiently small, we consider a function $\zeta \epsilon$ $C_{0}\left(B_{r_{0}}^{\infty}\left(P_{0}\right)\right)$ in such a way that $\zeta=1$ in $B_{r_{0} / 2}\left(P_{0}\right)$. We perform the same coordinate transformation as in the beginning of $\$ 3$. Then the bilinear form $B[u, v]$ is reduced to the form

$$
\begin{aligned}
\widetilde{B}_{\epsilon}[u, v]= & \epsilon\left\{\left(J E_{i} u, E_{i} v\right)+(J u, v)\right\}+\left(J a_{i j} \Phi E_{i} u, E_{j} v\right) \\
& +\left(J\left(a_{i j} \Phi_{x_{j}}+b_{i} \Phi\right) E_{i} u, v\right)+(J c \Phi u, v)+\int_{y=0} \beta j u v d x,\left({ }^{8}\right)
\end{aligned}
$$

where $E_{i}$ are differential operators of first order and $J, j$ are the functions produced by the coordinate transformation. From now on we put $\widetilde{B}_{\nu}=\widetilde{B}_{\epsilon \nu}$ simply.

Then for the solutions of (4.4) we have

Lemma 5.1. There is a constant $C$ independent of $\nu$ such that

$$
\begin{gathered}
\left|\widetilde{B}_{\nu}\left[D_{x}\left(\zeta u_{\nu}\right), D_{x}\left(\zeta u_{\nu}\right)\right]-\widetilde{B}_{\nu}\left[\zeta u_{\nu}, J^{-1} D_{x}^{*} J D_{x}\left(\zeta u_{\nu}\right)\right]\right| \\
\leq C\left(\left\|\zeta u_{\nu}\right\|_{1}\left(\epsilon_{\nu}\right)\left\|D_{x}\left(\zeta u_{\nu}\right)\right\|_{1}+\left\|\zeta u_{\nu}\right\|_{1}\right)
\end{gathered}
$$

where $D_{x}^{*}=-D_{x}$.

Proof. Hereafter the constants and the functions $\zeta u_{\nu}$ will be denoted simply $C$ and $v$, respectively. Then from (5.1) we see

$$
\begin{aligned}
\widetilde{B}_{\nu}\left[D_{x} v, D_{x} v\right]= & \epsilon_{\nu}\left(J E_{i} D_{x} v, E_{i} D_{x} v\right)+\epsilon_{\nu}\left(J D_{x} v, D_{x} v\right) \\
& +\left(J a_{i j} \Phi E_{i} D_{x} v, E_{j} D_{x} v\right)+\left(J\left(a_{i j} \Phi_{x_{j}}+b_{i} \Phi\right) E_{i} D_{x} v, D_{x} v\right) \\
& +\left(J c \Phi D_{x} v, D_{x} v\right)+\int_{y=0} \beta j D_{x} v \cdot D_{x} v d x \\
\equiv & \sum_{k=1}^{6} I_{k}, \text { say. }
\end{aligned}
$$

First we have

(8) The function $\Phi_{x_{j}}$ means the derivative of $\Phi$ with the original variable $x_{j}$. 


$$
\begin{aligned}
I_{1}= & \epsilon_{\nu}\left(\left(J E_{i} D_{x}-D_{x} J E_{i}\right) v, E_{i} D_{x} v\right) \\
& +\epsilon_{\nu}\left(J E_{i} v,\left(D_{x}^{*} E_{i}-E_{i} J^{-1} D_{x}^{*} J\right) D_{x} v\right)+\epsilon_{\nu}\left(J E_{i} \nu, E_{i} J^{-1} D_{x}^{*} J D_{x} v\right) .
\end{aligned}
$$

Hence we see immediately that

$$
\left|I_{1}-\epsilon_{\nu}\left(J E_{i} v, E_{i} J^{-1} D_{x}^{*} J D_{x} v\right)\right| \leq C \epsilon_{\mu}\|v\|_{1}\left\|D_{x} v\right\|_{1} .
$$

Quite similarly

$$
\left|I_{2}-\epsilon_{\nu}\left(J v, J^{-1} D_{x}^{*} J D_{x} v\right)\right| \leq C \epsilon_{\nu}\|\nu\|_{1}\left\|D_{x} v\right\|_{1} .
$$

Next let us examine $I_{3}$. Denoting $J a_{i j} \Phi$ simply by $a_{i j}^{\prime}$, we see

$$
\begin{aligned}
I_{3}= & \left(\left(a_{i j}^{\prime} E_{i} D_{x}-D_{x} a_{i j}^{\prime} E_{i}\right) v, E_{j} D_{x} v\right) \\
& +\left(a_{i j}^{\prime} E_{i} v,\left(D_{x}^{*} E_{j}-E_{j} J^{-1} D_{x}^{*} J\right) D_{x} v\right)+\left(a_{i j}^{\prime} E_{i} \nu, E_{j} J^{-1} D_{x}^{*} J D_{x} v\right) \\
& I_{3,1}+I_{3,2^{-}}+I_{3,3}, \text { say. }
\end{aligned}
$$

For $I_{3,1}$ we have

$$
a_{i j}^{\prime} E_{i} D_{x}-D_{x} a_{i j}^{\prime} E_{i}=a_{i j}^{\prime}\left(E_{i} D_{x}-D_{x} E_{i}\right)+\left(a_{i j}^{\prime} D_{x}-D_{x} a_{i j}^{\prime}\right) E_{i}
$$

and for $I_{3,2}$,

$$
\begin{aligned}
& \left(D_{x}^{*} E_{j}-E_{j} J^{-1} D_{x}^{*} J\right) D_{x} v \\
& \quad=\left(E_{j} D_{x}-D_{x} E_{j}\right) D_{x} v+E_{j}\left(J^{-1} J_{x}\right) \cdot D_{x} v+J^{-1} J_{x} E_{j} D_{x} v \cdot(9)
\end{aligned}
$$

From the above we have

$$
\begin{aligned}
I_{3,1}+I_{3,2}= & \left(a_{i j}^{\prime}\left(E_{i} D_{x}-D_{x} E_{i}\right) v, E_{j} D_{x} v\right)+\left(a_{i j}^{\prime} E_{i} v,\left(E_{j} D_{x}-D_{x} E_{j}\right) D_{x} v\right) \\
& -\left(\left(a_{i j}^{\prime}\right)_{x} E_{i} v, E_{j} D_{x} v\right)+\left(a_{i j}^{\prime} E_{i} v, E_{j}\left(J^{-1} J_{x}\right) \cdot D_{x} v\right) \\
& +\left(a_{i j}^{\prime} E_{i} v, J^{-1} J_{x} E_{j} D_{x} v\right) .
\end{aligned}
$$

Obviously the fourth term in the right-hand side is not greater than $C\|v\|_{1}^{2}$. By the fact $a_{i j}^{\prime}=a_{j i}^{\prime}$ and by integration by parts, the sum of the first and the second term in the right-hand side is estimated by $C\|v\|_{1}^{2}$ from the above. Similarly the same estimates hold also for the other terms in (5.7). Hence we get

$$
\left|I_{3}-\left(a_{i j}^{\prime} E_{i} \nu, E_{j} J^{-1} D_{x}^{*} J D_{x} v\right)\right| \leq C\|v\|_{1}^{2}
$$

In a similar manner as the above we easily obtain the following estimates:

(9) The letter $J_{x}$ means $D_{x} J$. 


$$
\begin{aligned}
&\left|I_{4}-\left(J\left(a_{i j} \Phi_{x_{j}}+b_{i} \Phi\right) E_{i} v, J^{-1} D_{x}^{*} J D_{x} v\right)\right|, \\
&\left|I_{5}-\left(J c \Phi v, J^{-1} D_{x}^{*} J D_{x} v\right)\right| \leq C\|v\|_{1}^{2}
\end{aligned}
$$

and

$$
\left|I_{6}-\int_{y=0} \beta j v J^{-1} D_{x}^{*} J D_{x} v d x\right| \leq C\left\|_{v}\right\|_{1}^{2}
$$

Combining (5.5), (5.6), (5.8), (5.9) and (5.10), we have finished the proof. Further we have

Lemma 5.2. It bolds that

$$
\left|\widetilde{B}_{\nu}\left[\zeta u_{\nu}, J^{-1} D_{x}^{*} J D_{x}\left(\zeta u_{\nu}\right)\right]-\widetilde{B}_{\nu}\left[u_{\nu}, \zeta J^{-1} D_{x}^{*} J D_{x}\left(\zeta u_{\nu}\right)\right]\right| \leq C\left\|u_{\nu}\right\|_{1}^{2},
$$

where $C$ is independent of $\nu$.

Proof. Put $C_{\nu}[u, v]=B_{\nu}[\zeta u, v]-B_{\nu}[u, \zeta v]$. Then we see

$$
C_{\nu}[u, v]=\epsilon_{\nu}\left\{\left(\zeta_{x_{i}} u, v_{x_{i}}\right)-\left(\zeta_{x_{i}}{ }^{u} x_{i}, v\right)\right\}+C[u, v]
$$

where

$$
C[u, v]=\left(a_{i j} \Phi \zeta_{x_{i}} u, v_{x_{j}}\right)-\left(a_{i j} \Phi \zeta_{x_{j}} u_{x_{i}}, v\right)+\left(\left(a_{i j} \Phi x_{j}+b{ }_{i} \Phi\right) \zeta_{x_{i}} u, v\right)
$$

Let $C[u, v]$ be the reduced form of $C[u, v]$ by the coordinate transformation, and we denote $\zeta u_{\nu}$ simply by $v$. Then (5.11) implies

$$
\begin{aligned}
\tilde{C}_{\nu}\left[u_{\nu}, J^{-1} D_{x}^{*} J D_{x} \nu\right]=\widetilde{B}_{\nu}\left[\nu, J^{-1} D_{x}^{*} J D_{x} \nu\right]-\widetilde{B}_{\nu}\left[u_{\nu}, \zeta J^{-1} D_{x}^{*} J D_{x} v\right] \\
=\epsilon_{\nu}\left(J \zeta_{x_{i}} u_{\nu}, E_{i} J^{-1} D_{x}^{*} J D_{x} v\right)-\epsilon_{\nu}\left(J \zeta_{x_{i}} E_{i} u_{\nu}, J^{-1} D_{x}^{*} J D_{x} v\right) \\
+\left(J a_{i j} \Phi \zeta_{x_{i}} u_{\nu}, E_{j} J^{-1} D_{x}^{*} J D_{x} \nu\right)-\left(J a_{i j} \Phi \zeta_{x_{j}} E_{i} u_{\nu}, J^{-1} D_{x}^{*} J D_{x} v\right) \\
\quad+\left(J\left(a_{i j} \Phi_{x_{j}}+b_{i} \Phi\right) \zeta_{x_{i}} u_{\nu}, J^{-1} D_{x}^{*} J D_{x} \nu\right) \\
\equiv \sum_{k=1}^{5} I_{k}, \text { say. }
\end{aligned}
$$

Here the suffix $x_{i}$ of $\zeta$ and $\Phi$ are the variables of the original coordinate.

Exchanging differential operators and integrating by parts, we get

$$
\left|I_{1}-\epsilon_{\nu}\left(\zeta_{x_{i}} D_{x} u_{\nu}, \zeta J D_{x} E_{i} u_{\nu}\right)\right| \leq C \epsilon_{\nu}\left\|u_{\nu}\right\|_{1}^{2}
$$

and

$$
\left|I_{2}+\epsilon_{\nu}\left(J \zeta_{x_{i}} D_{x} E_{i} u_{\nu}, \zeta D_{x} u_{\nu}\right)\right| \leq C \epsilon_{\nu}\left\|u_{\nu}\right\|_{1}^{2}
$$


Accordingly,

$$
\left|I_{1}+I_{2}\right| \leq C \epsilon_{\nu}\left\|u_{\nu}\right\|_{1}^{2}
$$

In a similar manner we see

$$
\left|I_{3}-\left(a_{i j} \Phi \zeta_{x_{i}} J D_{x}^{*} D_{x} u_{\nu}, \zeta E_{j} u_{\nu}\right)\right| \leq C\left\|u_{\nu}\right\|_{1}^{2}
$$

and

$$
\left|I_{4}+\left(a_{i j} \Phi \zeta_{x_{j}} J D_{x}^{*} D_{x} u_{\nu}, \zeta E_{i} u_{\nu}\right)\right| \leq C\left\|u_{\nu}\right\|_{1}^{2}
$$

Hence

$$
\left|I_{3}+I_{4}\right| \leq C\left\|u_{\nu}\right\|_{1}^{2}
$$

In addition the estimate

$$
\left|I_{s}\right| \leq C\left\|u_{\nu}\right\|_{1}^{2}
$$

is obvious. Combining (5.12) (5.15), we have obtained the lemma.

By Lemma 5.1 and (4.3) we have

$$
\begin{aligned}
& \epsilon_{\nu}\left\|D_{x}\left(\zeta u_{\nu}\right)\right\|_{1}^{2}+\left\|D_{x}\left(\zeta u_{\nu}\right)\right\|_{W}^{2} \\
& \quad \leq C\left(\left|\widetilde{B}_{\nu}\left[\zeta u_{\nu}, J^{-1} D_{x}^{*} J D_{x}\left(\zeta u_{\nu}\right)\right]\right|+\epsilon_{\nu}\left\|\zeta u_{\nu}\right\|_{1}\left\|D_{x}\left(\zeta u_{\nu}\right)\right\|_{1}+\left\|\zeta u_{\nu}\right\|_{1}^{2}\right) .
\end{aligned}
$$

Taking $\epsilon_{\nu}$ as sufficiently small we obtain

$$
\left\|D_{x}\left(\zeta u_{\nu}\right)\right\|_{W}^{2} \leq C\left(\left|\widetilde{B}_{\nu}\left[\zeta u_{\nu}, J^{-1} D_{x}^{*} J D_{x}\left(\zeta u_{\nu}\right)\right]\right|+\left\|\zeta u_{\nu}\right\|_{1}^{2}\right) .
$$

On the other hand it follows from Lemma 5.2 that

$$
\left|\widetilde{B}_{\nu}\left[\zeta u_{\nu}, J^{-1} D_{x}^{*} J D_{x}\left(\zeta u_{\nu}\right)\right]\right| \leq\left|\widetilde{B}_{\nu}\left[u_{\nu}, \zeta J^{-1} D_{x}^{*} J D_{x}\left(\zeta u_{\nu}\right)\right]\right|+C\left\|u_{\nu}\right\|_{1}^{2}
$$

and from (4.4) we have

$$
\tilde{B}_{\nu}\left[u_{\nu}, \zeta J^{-1} D_{x}^{*} J D_{x}\left(\zeta u_{\nu}\right)\right]=\left(J \Phi f_{\nu}, \zeta J^{-1} D_{x}^{*} J D_{x}\left(\zeta u_{\nu}\right)\right) .
$$

It is easy to see that

$$
\left.\left|\left(J \Phi f_{\nu}, \zeta J^{-1} D_{x}^{*} J D_{x}\left(\zeta u_{\nu}\right)\right)\right| \leq C\left\|f_{\nu}\right\|_{0} \| D_{x}\left(\zeta u_{\nu}\right)\right) \|_{1} \cdot
$$

Noting the boundedness of $\left\|I_{\nu}\right\|_{0}$, we have by $(5.16) \sim(5.19)$

$$
\left\|D_{x}\left(\zeta u_{\nu}\right)\right\|_{W}^{2} \leq C\left(\left\|u_{\nu}\right\|_{1}^{2}+\left\|D_{x}\left(\zeta u_{\nu}\right)\right\|_{W}\right) .
$$


Therefore we get

$$
\left\|D_{x}\left(\zeta u_{\nu}\right)\right\|_{W}^{2} \leq C\left(\left\|u_{\nu}\right\|_{1}^{2}+1\right)
$$

Now from Lemma 3.4 we see

$$
\begin{aligned}
\left\|\zeta u_{\nu}\right\|_{1}^{2} \leq C\{\delta & \left(\left\|\Phi^{1 / 2} D_{y}^{2}\left(\zeta u_{\nu}\right)\right\|^{2}+\left\|\Phi^{1 / 2} D_{y} D_{x}\left(\zeta u_{\nu}\right)\right\|^{2}\right) \\
& \left.+\delta^{-1}\left(\left\|\Phi^{1 / 2} D\left(\zeta u_{\nu}\right)\right\|^{2}+\left\|\Phi^{1 / 2}\left(\zeta u_{\nu}\right)\right\|^{2}\right)\right\}
\end{aligned}
$$

where $D$ is either $D_{x}$ or $D_{y}$. Let us note the following fact. Obviously

$$
D_{y}^{2}\left(\zeta u_{\nu}\right)=D_{y}^{2} \zeta \cdot u+2 D_{y} \zeta \cdot D_{y} u+\zeta D_{y}^{2} u_{\nu}
$$

and we see in the original coordinate

$$
-\epsilon_{\nu} \Delta u_{\nu}+\epsilon_{\nu} u_{\nu}+\Phi £ u=\Phi f_{\nu}
$$

because $u_{\nu}$ are solutions of (4.4)。 That is, $D_{y}^{2} u_{\nu}$ is a linear combination of $f_{\nu}$ $D_{y} D_{x} u_{\nu} D_{x}^{2} u_{\nu}, D u_{\nu}$ and $u_{\nu}$. Let $\left\{\zeta_{i}\right\}$ be a partition of unity for $\bar{\Omega}$ and $\Sigma_{i} \zeta_{i}^{2}=$ 1 in $\bar{\Omega}$. Then it is seen that $\left\|u_{\nu}\right\|_{1}^{2} \leq \Sigma_{i}\left\|\zeta_{i} u_{\nu}\right\|_{1}^{2}+C\left\|u_{\nu}\right\|_{0}^{2}$ and $\left\|u_{\nu}\right\|_{0}$ are bounded. Hence from (5.20), (5.21) and the above facts we get

$$
\sum_{i}\left\|D_{x}\left(\zeta_{i} u_{\nu}\right)\right\|_{W}^{2} \leq C \delta \sum_{i}\left\|D_{x}\left(\zeta_{i} u_{\nu}\right)\right\|_{W}^{2}+C \delta^{-1}
$$

Here let us take the positive number $\delta$ such as $C \delta<1 / 2$. Then we obtain $\Sigma_{i}\left\|D_{x}\left(\zeta_{i} u_{\nu}\right)\right\|_{w}^{2} \leq C$. Hence

$$
\left\|\Phi^{1 / 2} D_{x}^{2}\left(\zeta_{i} u_{\nu}\right)\right\|,\left\|\Phi^{1 / 2} D_{x} D_{y}\left(\zeta_{i} u_{\nu}\right)\right\| \leq C
$$

Since $D_{y}^{2} u_{\nu}$ is a linear combination of other terms in (5.22), it holds also that $\left\|\Phi^{1 / 2} D_{y}^{2}\left(\zeta_{i} u_{\nu}\right)\right\| \leq C$. Again applying Lemma 3.4, we can conclude that $\left\|u_{\nu}\right\|_{2}$ are bounded with respect to $\nu$. Thus we have completed the proof of Proposition 5.1.

6. In the previous section we have proved our theorem when $k=0$. From now on let us prove the theorem for general $k$. Since the argument in this section is almost parallel to the previous section, we shall proceed briefly.

For given $f \in V^{k}(\Omega)$ we take a sequence $\left\{f_{\nu}\right\}$ in $C^{\infty}(\bar{\Omega})$ satisfying $f_{\nu} \rightarrow f$ $(\nu \rightarrow \infty)$ in $V^{k}(\Omega)$. Let $u_{\nu}$ be the solutions of (4.4). Then, of course, $u_{\nu}$ are sufficiently smooth in $\bar{\Omega}$.

First generalizing Lemma 5.1, we have

Lemma 6.1. There is a constant $C$ such that 


$$
\begin{gathered}
\left|\widetilde{B}_{\nu}\left[D_{x}^{k+1}\left(\zeta u_{\nu}\right), D_{x}^{k+1}\left(\zeta u_{\nu}\right)\right]-\widetilde{B}_{\nu}\left[\zeta u_{\nu},\left(J^{-1} D_{x}^{*} J\right)^{k+1} D_{x}^{k+1}\left(\zeta u_{\nu}\right)\right]\right| \\
\leq C\left\|\zeta u_{\nu}\right\|_{k+1}\left(\epsilon_{\nu}\left\|D_{x}^{k+1}\left(\zeta u_{\nu}\right)\right\|_{1}+\left\|\zeta u_{\nu}\right\|_{k+1}\right) .
\end{gathered}
$$

Proof. It is easily seen that

$$
\begin{gathered}
\widetilde{B}_{\nu}\left[D_{x}^{k+1}\left(\zeta u_{\nu}\right), D_{x}^{k+1}\left(\zeta u_{\nu}\right)\right]-\widetilde{B}_{\nu}\left[\zeta u_{\nu},\left(J^{-1} D_{x}^{*} J\right)^{k+1} D_{x}^{k+1}\left(\zeta u_{\nu}\right)\right] \\
=\sum_{l=0}^{k}\left\{\widetilde{B}_{\nu}\left[D_{x}^{k+1}-l\left(\zeta u_{\nu}\right),\left(J^{-1} D_{x}^{*} J\right)^{l} D_{x}^{k+1}\left(\zeta u_{\nu}\right)\right]\right. \\
\left.\quad-\widetilde{B}_{\nu}\left[D_{x}^{k-l}\left(\zeta u_{\nu}\right),\left(J^{-1} D_{x}^{*} J\right)^{l+1} D_{x}^{k+1}\left(\zeta u_{\nu}\right)\right]\right\}
\end{gathered}
$$

Let us note each term in the right-hand side. Putting $\zeta u_{\nu}=v_{2}$ we see

$$
\begin{aligned}
\tilde{B}_{\nu}\left[D_{x}^{k+1-l}\left(\zeta u_{\nu}\right),\left(J^{-1} D_{x}^{*} J\right)^{l} D_{x}^{k+1}\left(\zeta u_{\nu}\right)\right] \\
=\epsilon_{\nu}\left(J E_{i} D_{x}^{k+1-l} v, E_{i}\left(J^{-1} D_{x}^{*} J\right)^{l} D_{x}^{k+1} v\right)+\epsilon_{\nu}\left(J D_{x}^{k+1-l} v,\left(J^{-1} D_{x}^{*} J\right) D_{x}^{k+1} v\right) \\
\quad+\left(J a_{i j} \Phi E_{i} D_{x}^{k+1-l} v, E_{j}\left(J^{-1} D_{x}^{*} J\right)^{l} D_{x}^{k+1} v\right) \\
\quad+\left(J\left(a_{i j} \Phi_{x}+b_{i} \Phi\right) E_{i} D_{x}^{k+1-l} v,\left(J^{-1} D_{x}^{*} J\right) D_{x}^{l+1} v\right) \\
\quad+\left(J c \Phi D_{x}^{k+1-l} v,\left(J^{-1} D_{x}^{*} J\right)^{l} D_{x}^{k+1} v\right) \\
\quad+\int_{y=0} \beta j D_{x}^{k+1-l} v^{k}\left(J^{-1} D_{x}^{*} J\right)^{l} D_{x}^{k+1} v d x \\
\equiv \sum_{k=1}^{6} l_{k}, \text { say. }
\end{aligned}
$$

By integration by parts we have

$$
\left|I_{1}-\epsilon_{\nu}\left(J E_{i} D_{x}^{k-l} v, E_{i}\left(J^{-1} D_{x}^{*} J\right)^{l+1} D_{x}^{k+1} v\right)\right| \leq C \epsilon_{\nu}\|v\|_{k+1}\left\|D_{x}^{k+1} v\right\|_{1}
$$

and

$$
\left|I_{2}-\epsilon_{\nu}\left(J D_{x}^{k-l} v,\left(J^{-1} D_{x}^{*} J\right)^{l+1} D_{x}^{k+1} v\right)\right| \leq C \epsilon_{\nu}\|v\|_{k+1}\left\|D_{x}^{k+1} v\right\|_{1} .
$$

Let us examine $I_{3}$ more carefully. Putting $a_{i j}^{\prime}=J a_{i j} \Phi$, we see

$$
\begin{aligned}
I_{3}= & \left(\left(a_{i j}^{\prime} E_{i} D_{x}-D_{x} a_{i j}^{\prime} E_{i}\right) D_{x}^{k-l} v, E_{j}\left(J^{-1} D_{x}^{*} J\right)^{l} D_{x}^{k+1} v\right) \\
& +\left(a_{i j}^{\prime} E_{i} D_{x}^{k-l} v,\left(D_{x}^{*} E_{j}-E_{j}\left(J^{-1} D_{x}^{*} J\right)\right)\left(J^{-1} D_{x}^{*} J\right) D_{x}^{k+1} v\right) \\
& +\left(a_{i j}^{\prime} E_{i} D_{x}^{k-l} v, E_{j}\left(J^{-1} D_{x}^{*} J\right)^{l+1} D_{x}^{k+1} v\right) \\
\equiv & I_{3,1}+I_{3,2}+I_{3,3}, \text { say. }
\end{aligned}
$$


Obviously

$$
a_{i j}^{\prime} E_{i} D_{x}-D_{x} a_{i j}^{\prime} E_{i}=a_{i j}^{\prime}\left(E_{i} D_{x}-D_{x} E_{i}\right)+\left(a_{i j}^{\prime} D_{x}-D_{x} a_{i j}^{\prime}\right) E_{i}
$$

and

$$
D_{x}^{*} E_{j}-E_{j} J^{-1} D_{x}^{*} J=\left(E_{j} D_{x}-D_{x} E_{j}\right)+E_{j}\left(J^{-1} J_{x}\right)+J^{-1} J_{x} E_{j}
$$

Hence we have

$$
\begin{aligned}
l_{3,1}+I_{3,2}= & \left(a_{i j}^{\prime}\left(E_{i} D_{x}-D_{x} E_{i}\right) D_{x}^{k-l} v, E_{j}\left(J^{-1} D_{x}^{*} J\right)^{l} D_{x}^{k+1} v\right) \\
& +\left(a_{i j}^{\prime} E_{i} D_{x}^{k-l} v,\left(E_{j} D_{x}-D_{x} E_{j}\right)\left(J^{-1} D_{x}^{*} J\right) D_{x}^{k+1} v\right) \\
& -\left(\left(a_{i j}^{\prime}\right)_{x} E_{i} D_{x}^{k-l} v, E_{j}\left(J^{-1} D_{x}^{*} J\right)^{l} D_{x}^{k+1} v\right) \\
& +\left(a_{i j}^{\prime} E_{i} D_{x}^{k-l} v, E_{j}\left(J^{-1} J_{x}\right) \cdot\left(J^{-1} D_{x}^{*} J\right)^{l} D_{x}^{k+1} v\right) \\
& +\left(a_{i j}^{\prime} E_{i} D_{x}^{k-l} v, J^{-1} J_{x} E_{j}\left(J^{-1} D_{x}^{*} J\right)^{l} D_{x}^{k+1} v\right)
\end{aligned}
$$

Thus in the same manner as getting (5.8) from (5.7), we obtain

$$
\left|I_{3}-\left(a_{i j}^{\prime} E_{i} D_{x}^{k-l} v, E_{j}\left(J^{-1} D_{x}^{*} J\right)^{l+1} D_{x}^{k+1} v\right)\right| \leq C\|v\|_{k+1}^{2} .
$$

Further the following estimates are obvious:

$$
\begin{aligned}
\left|I_{4}-\left(J\left(a_{i j} \Phi_{x_{j}}+b_{i} \Phi\right) E_{i} D_{x}^{k-l} v,\left(J^{-1} D_{x}^{*} J\right)^{l+1} D_{x}^{k+1} v\right)\right|, \\
\left|I_{5}-\left(J c \Phi D_{x}^{k-l} v,\left(J^{-1} D_{x}^{*} J\right)^{l+1} D_{x}^{k+1} v\right)\right| \leq C\|v\|_{k+1}^{2}
\end{aligned}
$$

and

$$
\left|I_{6}-\int_{y=0} \beta j D_{x}^{k-l} v\left(J^{-1} D_{x}^{*} J\right)^{l+1} D_{x}^{k+1} v d x\right| \leq C\|v\|_{k+1}^{2} .
$$

Hence from (6.1), (6.2) and by all the above estimates we have obtained Lemma 6.1.

Secondly in parallel with Lemma 5.2 we have

Lemma 6.2. It bolds that

$\left|\tilde{B}_{\nu}\left[\zeta u_{\nu},\left(J^{-1} D_{x}^{*} J\right)^{k+1} D_{x}^{k+1}\left(\zeta u_{\nu}\right)\right]-\widetilde{B}_{\nu}\left[u_{\nu}, \zeta\left(J^{-1} D_{x}^{*} J\right)^{k+1} D_{x}^{k+1}\left(\zeta u_{\nu}\right)\right]\right| \leq C\left\|u_{\nu}\right\|_{k+1}^{2} \cdot$

Proof. Obviously we see 


$$
\begin{aligned}
\tilde{B}_{\nu} & {\left[\zeta u_{\nu},\left(J^{-1} D_{x}^{*} J\right)^{k+1} D_{x}^{k+1}\left(\zeta u_{\nu}\right)\right]-\tilde{B}_{\nu}\left[u_{\nu}, \zeta\left(J^{-1} D_{x}^{*} J\right)^{k+1} D_{x}^{k+1}\left(\zeta u_{\nu}\right)\right] } \\
= & \epsilon_{\nu}\left\{\left(J E_{i} \zeta u_{\nu}, E_{i}\left(J^{-1} D_{x}^{*} J\right)^{k+1} D_{x}^{k+1}\left(\zeta u_{\nu}\right)\right)-\left(J E_{i} u_{\nu}, E_{i} \zeta\left(J^{-1} D_{x}^{*} J\right)^{k+1} D_{x}^{k+1}\left(\zeta u_{\nu}\right)\right)\right\} \\
& +\left\{\left(J a_{i j} \Phi E_{i}\left(\zeta u_{\nu}\right), E_{j}\left(J^{-1} D_{x}^{*} J\right)^{k+1} D_{x}^{k+1}\left(\zeta u_{\nu}\right)\right)\right.
\end{aligned}
$$

$$
\begin{array}{r}
\left.-\left(J a_{i j} \Phi E_{i} u_{\nu}, E_{j} \zeta\left(J^{-1} D_{x}^{*} J\right)^{k+1} D_{x}^{k+1}\left(\zeta u_{\nu}\right)\right)\right\} \\
+\left\{\left(J\left(a_{i j} \Phi_{x_{j}}+b_{i} \Phi\right) E_{i} \zeta u_{\nu},\left(J^{-1} D_{x}^{*} J\right)^{k+1} D_{x}^{k+1}\left(\zeta u_{\nu}\right)\right)\right. \\
\left.-\left(J\left(a_{i j} \Phi_{x_{j}}+b_{i} \Phi\right) E_{i} u_{\nu}, \zeta\left(J^{-1} D_{x}^{*} J\right)^{k+1} D_{x}^{k+1}\left(\zeta u_{\nu}\right)\right)\right\} .
\end{array}
$$

It is easily seen that the first and the third terms are estimated from the above by $C\left\|u_{\nu}\right\|_{k+1}^{2}$. The remaining term (the second term) equals

$$
\begin{aligned}
\left\{\left(J a_{i j} \Phi \zeta_{x_{i}} u_{\nu},\right.\right. & \left.E_{j}\left(J^{-1} D_{x}^{*} J\right)^{k+1} D_{x}^{k+1}\left(\zeta u_{\nu}\right)\right) \\
& \left.-\left(J a_{i j} \Phi E_{i} u_{\nu}, \zeta_{x_{j}}\left(J^{-1} D_{x}^{*} J\right)^{k+1} D_{x}^{k+1}\left(\zeta u_{\nu}\right)\right)\right\} \\
= & \left\{\left(J a_{i j} \Phi \zeta_{x_{i}} u_{\nu}, E_{j}\left(J^{-1} D_{x}^{*} J\right)^{k+1} D_{x}^{k+1}\left(\zeta u_{\nu}\right)\right)\right. \\
& \left.-\left(J a_{i j} \Phi \zeta_{x_{i}}{ }_{\nu}, \zeta\left(J^{-1} D_{x}^{*} J\right)^{k+1} D_{x}^{k+1} E_{j} u_{\nu}\right)\right\} \\
+ & \left\{\left(J a_{i j} \Phi \zeta_{x_{i}} u_{\nu}, \zeta\left(J^{-1} D_{x}^{*} J\right)^{k+1} D_{x}^{k+1} E_{j} u_{\nu}\right)\right. \\
& \left.\quad\left(J a_{i j} \Phi \zeta_{x_{i}}\left(D_{x}^{*}\right)^{k+1}\left(J^{-1} D_{x}^{*} J\right)^{k+1}\left(\zeta u_{\nu}\right), E_{j} u_{\nu}\right)\right\} \\
+ & \left\{\left(J a_{i j} \Phi \zeta_{x_{i}}\left(D_{x}^{*}\right)^{k+1}\left(J^{-1} D_{x}^{*} J\right)^{k+1}\left(\zeta_{\nu}\right), E_{j} u_{\nu}\right)\right. \\
& \left.\quad-\left(J a_{i j} \Phi E_{i} u_{\nu}, \zeta_{x_{j}}\left(J^{-1} D_{x}^{*} J\right)^{k+1} D_{x}^{k+1}\left(\zeta_{\nu}\right)\right)\right\} .
\end{aligned}
$$

Exchanging differential operators and integrating by parts for each term, we see that the second term on the right of (6.3) is estimated by $C\left\|u_{\nu}\right\|_{k+2}^{2}$ from the above. Thus we have completed the proof.

From the above lemmas we shall proceed along the argument of the previous section. By (4.4) we see

$$
\begin{aligned}
\widetilde{B}_{\nu}\left[u_{\nu}, \zeta\left(J^{-1} D_{x}^{*} J\right)^{k+1} D_{x}^{k+1}\left(\zeta u_{\nu}\right)\right] & =\left(J \Phi f_{\nu}, \zeta\left(J^{-1} D_{x}^{*} J\right)^{k+1} D_{x}^{k+1}\left(\zeta u_{\nu}\right)\right) \\
& =\left(\left(J D_{x} J^{-1}\right)^{k}\left(\zeta J \Phi f_{\nu}\right),\left(J^{-1} D_{x}^{*} J\right) D_{x}^{k+1}\left(\zeta u_{\nu}\right)\right)
\end{aligned}
$$


From Leibnitz' formula

Accordingly by (6.4) we see

$$
\left(J D_{x} J^{-1}\right)^{k}\left(\zeta J \Phi f_{\nu}\right)=\sum_{p+q \leq k} c_{p, q} D_{x}^{p} \Phi D_{x}^{q} f_{\nu}
$$

$$
\begin{aligned}
& \widetilde{B}_{\nu}\left[u_{\nu}, \zeta\left(J^{-1} D_{x}^{*} J\right)^{k+1} D_{x}^{k+1}\left(\zeta u_{\nu}\right)\right] \\
& \quad=\sum_{p+q \leq k}\left(c_{p, q} D_{x}^{p} \Phi D_{x}^{q} f_{\nu},\left(J^{-1} D_{x}^{*} J\right) D_{x}^{k+1}\left(\zeta u_{\nu}\right)\right) \equiv I_{p, q}, \text { say. }
\end{aligned}
$$

Since $p=0$ for $q=k$ and $p \leq 1$ for $q=k-1$, respectively, we get

$$
\left|I_{0, k}\right| \leq C\left\|f_{\nu}\right\|_{k}\left\|D_{x}^{k+1}\left(\zeta u_{\nu}\right)\right\|_{W}
$$

and

$$
\begin{aligned}
\| I_{1, q} \mid & \leq C\left\|f_{\nu}\right\|_{k-1}\left(\left\|\Phi_{x}\left(J^{-1} D_{x}^{*} J\right) D_{x}^{k+1}\left(\zeta u_{\nu}\right)\right\|+\left\|\Phi\left(J^{-1} D_{x}^{*} J\right) D_{x}^{k+1}\left(\zeta u_{\nu}\right)\right\|\right) \\
& \leq C\left\|f_{\nu}\right\|_{k-1}\left\|D_{x}^{k+1}\left(\zeta u_{\nu}\right)\right\|_{W} \quad \text { (by Lemma 3.3). }
\end{aligned}
$$

When $q \leq k-2$, it holds by integration by parts

$$
\left|I_{p, q}\right| \leq C\left\|f_{\nu}\right\|_{k-1}\left\|D_{x}^{k+1}\left(\zeta u_{\nu}\right)\right\| \text {. }
$$

Combining (6.5) (6.8), we obtain

$$
\left.\mid \tilde{B}_{\nu}\left[u_{\nu}, \zeta\left(J^{-1} D_{x}^{*}\right]\right)^{k+1} D_{x}^{k+1}\left(\zeta u_{\nu}\right)\right] \mid \leq C\left\|f_{\nu}\right\|_{k}\left\|D_{x}^{k+1}\left(\zeta u_{\nu}\right)\right\|_{W^{\bullet}}
$$

Taking $\epsilon_{\nu}$ as sufficiently small, we have, by Lemmas 6.1, 6.2 and (4.3),

$$
\left\|D_{x}^{k+1}\left(\zeta u_{\nu}\right)\right\|_{W}^{2} \leq C\left(\left\|u_{\nu}\right\|_{k+1}^{2}+1\right)
$$

Applying Lemma 3.4 , let us proceed in parallel with $\S 5$. Then finally we obtain

$$
\left\|\Phi^{1 / 2} D^{k+2}\left(\zeta u_{\nu}\right)\right\| \leq C .
$$

Hence $\left\|u_{\nu}\right\|_{k+2}$ are bounded with respect to $\nu$. This means that the function $u$ satisfying $u_{\nu} \rightarrow u$ in the weak sense is in $V^{k+2}(\Omega)$. Thus we have completed the theorem.

\section{REFERENCES}

1. M. S. Baouendi and C. Goulaouic, Régularité et théorie spectrale pour une class d'opérateurs elliptiques dégénérés, Arch. Rational Mech. Anal. 34 (1969), 361-378. MR 40 \#3085.

2. R. Courant, Methods of mathematical physics. Vol. II: Partial differential equations, Interscience, New York, 1962. MR 25 \#4216. 
3. S. Itô, Fundamental solutions of parabolic differential equations and boundary value problems, Japan. J. Math. 27 (1957), 55-102. MR 20 \#4702.

4. J.-L. Lions and E. Magenes, Problèmes aux limites non homogènes. II, Ann. Inst. Fourier (Grenoble) 11 (1961), 137-178. MR $26 \# 4047$.

5. T. Matsuzawa, Sur les équations $u_{t t}+t a_{u_{x x}}=f(a \geq 0)$, Nagoya Math. J. 42 (1971), 43-55. MR $44 \# 1923$.

6. V. G. Maz' ${ }^{j a}$, The degenerate problem with oblique derivative, Uspehi Mat. Nauk 25 (1970), 275-276. (Russian) MR $41 \# 4031$.

7. L. Nirenberg, Remarks on strongly elliptic partial differential equations, Comm. Pure Appl. Math. 8 (1955), 649-675. MR 17, 742.

8. N. Shimakura, Problèmes aux limites généraux du type elliptique dégénéré, J. Math. Kyoto Univ. 9 (1969), 275-335. MR 40 \#7628.

9. M. I. Višik and V. V. Grusin, Elliptic boundary value problems degenerating on a submanifold of the boundary, Dokl. Akad. Nauk SSSR 190 (1970), 255-258 = Soviet Math. Dokl. 11 (1970), 60-64. MR 41 \#7291.

DEPARTMENT OF MATHEMATICS, COLLEGE OF GENERAL EDUCATION, NAGOYA UNIVERSITY, CHIKUSA-KU, NAGOYA, JAPAN 